\title{
Introduction: Abstracts from the 25th Anniversary International CyberPsychology, CyberTherapy \& Social Networking Conference
}

\begin{abstract}
A S THE CONFERENCE CO-CHAIRS Professor Brenda K. Wiederhold and Professor Giuseppe Riva, it is our pleasure to invite you to explore the abstracts that were submitted and accepted for presentation at the 25th Anniversary International CyberPsychology, CyberTherapy \& Social Networking Conference (CYPSY25). CYPSY25 should have taken place from Monday June 22 to Wednesday June 24, 2020, at Università Cattolica del Sacro Cuore in Milan, Italy. Jointly organized by the Interactive Media Institute and Istituto Auxologico Italiano, with the support of Università Cattolica del Sacro Cuore and the European project AffecTech, the CYPSY conference series is the premier international conference on virtual reality and cyberpsychology. Attracting individuals from different disciplines, cultures, and backgrounds-researchers, designers, clinicians, developers, policymakers, and funding agents-our group has become one common community, with the shared purpose of creating technologies that will improve the health and well-being of all citizens, and exploring how technologies impact us, both societally and individually.

After careful and significant deliberation, on March 20, 2020, we made the very difficult decision to postpone the planned 25th anniversary conference. News from the World Health Organization and Centers for Disease Control and Prevention, along with both National and European governments, all voiced concern over the gathering of so many people in Lombardy, the region where Milan is located. The conference co-chairs, local committee members, and scientific committee deliberated over many days as we considered the many relevant factors. Many months go into planning our annual conference, and we had the commitment of a stellar group of speakers and presenters for a very high-quality program. We look forward each year to the opportunity to bring cyberpsychologists together for networking and continued learning. Ultimately though, with the mounting evidence about the seriousness of the public health crisis, we of course chose to place the highest priority on the health and safety of our members, speakers, participants, and staff.

We remain committed to providing quality education and research support for our members and the broader cyberpsychology community. With this in mind, we reached out to Mary Ann Liebert, Inc., publisher of the CYPSY conference's official journal, Cyberpsychology, Behavior and Social Networking. A supporter of CYPSY since its inception, the decision was made to publish the abstracts that had been accepted for the conference, so that our community's work would not go unnoticed for this year.

We thank the journal and publisher for this important contribution.

We are also pleased to announce that the conference has been rescheduled for June 14-16, 2021 (https:// www.interactivemediainstitute.com/cypsy25/)! We will keep you informed of new events and programs as 2020 progresses as well.

We send our best wishes for everyone's health and well-being during this difficult time, and we look forward to welcoming you to Milan in 2021!

Prof. Giuseppe Riva

Prof. Brenda K. Wiederhold

\author{
Abstracts may be accessed online at: \\ https://www.liebertpub.com/doi/suppl/10.1089/cyber.2020.29198.cypsy
}

